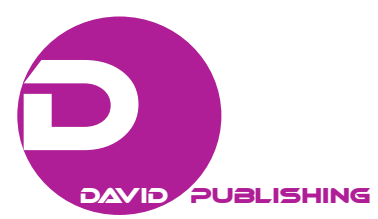

\title{
Existence Results of Abstract Impulsive Integrodifferential Systems with Measure of Non-compactness
}

\author{
K. Malar \\ Department of Mathematics, Erode Arts and Science College, Erode-638009, Tamil Nadu, INDIA. \\ A. Anguraj \\ Department of Mathematics, PSG College of Arts and Science, Coimbatore- 641 014, Tamil Nadu, INDIA.
}

\begin{abstract}
In this Paper, we study the existence of solutions for the nonlocal integrodifferential equations with interval impulse and measure of non compactness by using Mönch - fixed point theorem. Finally, an example is given to illustrate our main result.
\end{abstract}

Keywords: Integrodifferential equations, Interval impulse, Semigroup, Fixed point theorem, Measure of noncompactness.

\section{Introduction}

In this present paper, we are concerned with the existence of mild solutions are proved for a new class of non-instantaneous impulsive integrodifferential equations with nonlocal conditions:

$$
\begin{gathered}
\frac{d u(t)}{d t}=A u(t)+f\left(t, u(t), \int_{0}^{t} \rho(t, s) h(t, s, u(s)) d s\right), t \in\left(s_{i}, t_{i+1}\right], \quad i=0,1, \ldots, m \\
u(t)=g_{i}(t, u(t)), \quad t \in\left(t_{i}, s_{i}\right], \quad i=1,2, \ldots, m \\
u(0)=u_{0}+k(u)
\end{gathered}
$$

where $A$ generates a $C_{0}$ - semi group of bounded linear operator $T(t), t \geq 0$ defined on a Banach space $(X,\|\|$.$) .$ $u_{0} \in X, 0=t_{0}=s_{0}<t_{1} \leq s_{1} \leq t_{2}<\ldots<t_{m} \leq s_{m} \leq t_{m+1}=b$ are pre - fixed numbers, $k: X \rightarrow X$, and $g_{i} \in C\left(\left(t_{i}, s_{i}\right] \times X ; X\right)$ for all $i=1,2, \ldots, m, f$ is a given function $f:[0, b] \times X \times X \rightarrow X$ and $h \in C\left(D, R^{+}\right], D=\{(t, s) \mid s, t \in[0, b], t \geq s\}, R^{+}=[0,+\infty)$.

The main techniques relay on the impulsive integro - differential equations, Mönch fixed point theorem via measure of noncompactness.

The theory of semi group of bounded linear operator is closely related to the solution of differential and integrodifferential equations in Banach spaces. In recent years, this theory has been applied to a large class of nonlinear differential equations in Banach spaces. We refer to the papers [1-4] and the references cited therein. The method of semi group, existence and uniqueness of mild solutions of semi linear evolution equations were discussed by Pazy [5]. 
Recently, the theory of impulsive differential equations has become an important area of investigations, because of the wide possibilities for their applications in various fields of science and technology as theoretical physics, population dynamics, economics and so forth. Subsequently, many authors have investigated the existence solutions to impulsive differential equations or impulsive integrodifferential equations with their applications in Banach spaces; see [6-8] and the references therein.

In recent paper [9], Lan and Cui studied a class of initial value problems of nonlinear first order implicit impulsive integrodifferential equations in Banach space. By using the Mönch - fixed point theorem, they obtained some new existence theorem of solution for this class of nonlinear first order implicit impulsive integrodifferential equations in Banach spaces under some weaker conditions. For further details, see [1,7,10-13]. Shaochu, J., Gang. L [14] investigated a unified approach to nonlocal impulsive differential equations with measure of non-compactness. In [7], Ahmad et al. studies nonlocal problem of impulsive integrodifferential equations with measure of non-compactness by applying a new fixed point theorem. Eduardo Hernandez and Donal O' Regan [15] established on a new class of abstract impulsive differential equations for which the impulses are not instantaneous. In [16], JinRong Wang et al also proved periodic BVP integer/fractional order nonlinear differential equations with non - instantaneous impulses.

Motivated and inspired by the above works, we discuss the existence of mild solutions of (1.1) - (1.3) combining impulsive and nonlocal conditions. Our results are derived by using Mönch - fixed point theorem and Hausdroff measure of non compactness.

This paper is organized as follows:

In section 2, we introduce some definitions and notations and some preliminary notions. In section 3 , we present out main results on existence of solutions of the problem above and Example is presented in section 4 illustrating the applicability of the improved conditions.

\section{Preliminaries}

Let $\mathrm{C}([0, \mathrm{~b}] ; \mathrm{X})$ denote the Banach space of all continuous functions from $[0, b]$ into $X$ with the norm $\|\mathrm{u}\|_{\mathrm{C}}:=\sup \{|\mathrm{u}(\mathrm{t})|: t \in[0, b]\}$ for $u \in C([0, b] ; X) . A C_{\mathrm{o}}-\operatorname{semi}$ group $\mathrm{T}(\mathrm{t})$ is said to be compact if $\mathrm{T}(t)$ is compact for $t>0$. If the semi group $\mathrm{T}(t)$ is compact, then $t \rightarrow T(t) u$ are equicontinuous at all $t>0$ with respect to $\mathrm{u}$ in all bounded subset of $X$, that is, the semi group $\mathrm{T}(t)$ is equicontinuous.

Now we consider the space $P C([0, b] ; X):=\left\{u:[0, b] \rightarrow X: u \in C\left(\left(t_{k}, t_{k+1}\right], X\right), k=0,1, \ldots, m\right.$ and there exist $u\left(t_{k}^{-}\right)$and $u\left(t_{k}^{+}\right), k=1,2, . ., \mathrm{m}$, with $\left.u\left(t_{k}^{-}\right)=u\left(t_{k}\right)\right\}$ with the norm $\|u\|_{P C}:=\sup \{|u(t)|: t \in[0, b]\} \quad$. Set $\quad P C^{1}([0, b], X):=\left\{u \in P C([0, b], X): u^{\prime} \in P C([0, b], X) \quad\right.$ with $\|u\|_{P C^{1}}: \max \left\{\|u\|_{P C},\left\|u^{\prime}\right\|_{P c}\right\}$. Clearly, $P C^{1}([0, b], X)$ endowed with the norm $\|\cdot\|_{P C^{1}}$ is a Banach space.

In this paper, $\chi$ denotes the Hausdroff measure of noncompactness on both $X$ and $P C([0, b], X)$. The following lemma describes some properties of the Hausdroff measure of noncompactness.

Lemma 2.1: ([17]). Let $Y$ be a real Banach space $B, C \subseteq Y$ be bounded. Then

(1) $\mathrm{B}$ is pre-compact if and only if $\beta_{X}(B)=0$;

(2) $\beta_{Y}(B)=\beta_{Y}(\bar{B})=\beta_{Y}(\operatorname{conv} B)$, where $\bar{B}$ and conv $B$ mean the closure and convex hull of $B$ respectively. 
(3) $\beta_{Y}(B) \leq \beta_{Y}(C)$, where $B \subseteq C$;

(4) $\beta_{Y}(B+C) \leq \beta_{Y}(B)+\beta_{Y}(C)$, where $B+C=\{x+y: x \in B, y \in C\}$;

(5) $\beta_{Y}(B \cup C) \leq \max \left\{\beta_{Y}(B), \beta_{Y}(C)\right\}$;

(6) $\beta_{Y}(\lambda B) \leq|\lambda| \beta_{Y}(B)$ for any $\lambda \in \mathbb{R}$;

(7) If the map $Q: D(Q) \subseteq Y \rightarrow \mathbb{Z}$ is Lipchitz continuous with constant $\mathrm{k}$, then $\beta_{\mathbb{Z}}(Q B) \leq k \beta_{Y}(B)$ for any bounded subset $B \subseteq D(Q)$, where $\mathbb{Z}$ be a Banach space;

(8) $\beta_{Y}(B)=\inf \left\{d_{Y}(B, C) ; C \subseteq Y\right.$ is precompact $\}=\inf \left\{d_{Y}(B, C): C \subseteq Y\right.$ is finite valued $\}$, where $d_{Y}(B, C)$ means the non-symmetric (or symmetric) Hausdroff distance between $B$ and $C$ in $Y$;

(9) If $\left\{W_{n}\right\}_{n=1}^{+\infty}$ is decreasing sequence of bounded closed nonempty subsets of $Y$ and $\lim _{n \rightarrow \infty} \beta_{\mathrm{Y}}\left(\mathrm{W}_{n}\right)=0$, then $\bigcap_{n=1}^{+\infty} W_{n}$ is nonempty and compact in $Y$.

The map $Q: W \subseteq \mathrm{Y} \rightarrow \mathrm{Y}$ is said to be a $\beta_{\mathrm{Y}}$-contraction if there exists a constant $0<k<1$ such that $\beta_{\mathrm{Y}}(Q(B)) \leq k \beta_{\mathrm{Y}}(B)$ for any bounded closed subset $B \subseteq W$, where $Y$ is a Banach space.

Lemma 2.2: ([17]). If $W \subseteq P C \quad([0, K] ; X)$ is bounded, then $\beta(W(t)) \leq \beta_{\mathrm{PC}}(W)$ for all $t \in[0, K]$, where $W(t)=\{u(t): u \in W\} \subseteq X$. Furthermore if $W$ is equicontinuous on $[0, K]$, then $\beta(W(t))$ is continuous on $[0, K]$, and $\beta_{\mathrm{PC}}(W)=\sup \{\beta(W(t)): t \in[0, K]\}$.

Lemma 2.3: ([18]). If $\left\{u_{n}\right\}_{n=1}^{\infty} \subset L^{1}(0, K ; X)$ is uniformly integrable, then $\beta\left(\left\{u_{n}(t)\right\}_{n=1}^{\infty}\right)$ is measurable and

$$
\beta\left(\left\{\int_{0}^{t} u_{n}(s) d s\right\}_{n=1}^{\infty}\right) \leq 2 \int_{0}^{t} \beta\left\{u_{n}(s)\right\}_{n=1}^{\infty} d s
$$

Lemma 2.4: ([19]). If the semi group $\mathrm{T}(\mathrm{t})$ is equicontinuous and $\eta \in L^{1}\left(0, K ; R^{+}\right)$, then the set

$$
\left\{t \rightarrow \int_{0}^{t} T(t-s) u(s) d s ; u \in L^{1}\left(0, K ; R^{+}\right),\|u(s)\| \leq \eta(s) \text {, for a.e } s \in[0, K]\right\}
$$

Lemma 2.5: ([20)]. If $W$ is bounded, then for each $\varepsilon>0$, there is a sequence $\left\{u_{n}\right\}_{n=1}^{\infty} \subseteq W$ such that $\beta(W) \leq 2 \beta\left(\left\{u_{n}\right\}_{n=1}^{\infty}\right)+\varepsilon$.

The following fixed point theorem, a nonlinear alternative of Mönch type, plays a key role of the system (1.1)-(1.3).

Theorem 2.6: ([18]). Let $D$ be a closed convex subset of a Banach space $X$ and $0 \in D$. Assume that $F: D \rightarrow X$ is a continuous map which satisfies Mönch's condition, that is, $(M \subseteq D$ is countable, $M \subseteq \overline{c o}(\{0\} \cup F(M)) \rightarrow \bar{M}$ is compact). Then $\mathrm{F}$ has a fixed point in $D$.

Definition 2.1: A function $u:[0, b] \rightarrow X$ is called a mild solution of system (1.1) - (1.3) if $u \in P C([0, b], X)$ satisfies the following equation 
Existence Results of Abstract Impulsive Integrodifferential Systems with Measure of

$$
u(t)=\left\{\begin{array}{l}
T(t)\left[u_{0}-k(u)\right]+\int_{0}^{t} T(t-s) f\left(s, u(s), \int_{0}^{s} \rho(s, \tau) h(s, \tau, u(\tau)) d \tau\right) d s, t \in\left[0, t_{1}\right] \\
g_{i}(t, u(t)), t \in\left(t_{i}, s_{i}\right], i=1,2, \ldots, m, \\
T\left(t-s_{i}\right) g_{i}\left(s_{i}, u\left(s_{i}\right)\right)+\int_{s_{i}}^{t} T(t-s) f\left(s, u(s), \int_{0}^{s} \rho(s, \tau) h(s, \tau, u(\tau) d \tau) d s, t \in\left(s_{i}, t_{i+1}\right], i=0,1, \ldots, m\right.
\end{array}\right.
$$

\section{The Main Results}

In this section, we see the existence of solutions for problem (1.1) - (1.3) by applying theorem 2.6. For some real constants $r$, we define

$$
W=\left\{u \in P C([0, b] ; X),\|u(t)\|_{P C} \leq r, \forall t \in[0, b]\right\}
$$

Now we introduce the following hypotheses:

$\left(\mathrm{H}_{1}\right)$ The $C_{0}$-semi group $\mathrm{T}(t)$ generated by $A$ is equicontinuous and $N=\sup \{\|T(t)\|: t \in[0, b]\}$.

$\left(\mathrm{H}_{2}\right)(\mathrm{i})$ The functions $\mathrm{g}_{\mathrm{i}}$ are continuous and there are positive constants $L_{g i}$ such that $\left\|g_{i}(t, x)-g_{i}(t, y)\right\| \leq L_{g_{i}}[\|x-y\|]$ for all $x, y \in X, t \in\left(t_{i}, s_{i}\right]$ and each $i=0,1, \ldots, m$

(ii) There are positive constants $M_{i}$ such that $\left\|g_{i}(t, x)\right\| \leq M_{i}\|u\|$ for all $x \in X, t \in\left(t_{i}, s_{i}\right]$ and each $i=0$, $1, \ldots, m$.

(iii) For each bounded subset $B \subset X$ we have $\chi\left(g_{i}(t, B)\right) \leq M_{i}\left[\sup _{-\infty<s_{i} \leq 0} \chi\left(B\left(s_{i}\right)\right], i=1,2, \ldots, m\right.$.

$\left(\mathrm{H}_{3}\right)(\mathrm{i})$ There exists a function $m_{f} \in C([0, b] ; X)$ and a non-decreasing continuous function $\Omega_{f}: X \rightarrow X$ such that $\|f(t, u, v)\| \leq m_{f}(t) \Omega_{f}(\|u\|+\|v\|)$ for all $u \in X$ a.e $t \in[0, b]$.

(ii) There is an integrable function $\eta:[0, b] \rightarrow[0,+\infty]$ such that $\chi\left(f\left(t, D_{1}, D_{2}\right)\right) \leq \eta(t)\left[\sup _{-\infty<\theta \leq 0} \chi\left(D_{1}(\theta)\right)+\chi\left(D_{2}\right)\right]$ for a.e $t \in[0, b]$. and any bounded subsets $D_{1}, D_{2} \subset X$ and $\chi$ is the Hausdroff measure of noncompactness. Here we let $\int_{0}^{t} \eta(s) d s \leq \zeta^{*}$.

$\left(\mathrm{H}_{4}\right) \quad k: X \rightarrow X$ is continuous and there exists positive constants $\mathrm{c}$ and $\mathrm{d}$ such that $\|k(u)-k(v)\| \leq c\|u-v\|$ and $\|k(u)\| \leq c\|u\|+d$, for all $u \in P C(X)$.

$\left(\mathrm{H}_{5}\right) \quad f:[0, b] \times X \times X \rightarrow X$ is of caratheodory type, that is $f(., u, G u)$ is measurable for all $u \in X$ and $f(t, . .$.$) is continuous for a.e t \in[0, b]$.

$\left(\mathrm{H}_{6}\right)$ (i) The function $h(t, s,):. X \rightarrow X$ is continuous for a.e, $(t, s) \in \Delta$, and for each $u \in X$, the function $h(., ., u): \Delta \rightarrow X$ is measurable. Moreover, there exists a function $v: \Delta \rightarrow R^{+}$with $\sup _{t \in[0, b]} \int_{0}^{t} v(t, s) d s:=v^{*}<\infty$ such that $\|h(t, s, u)\| \leq v(t, s)\|u\|, u \in X$

(ii) For any bounded set $D_{1} \subset X$ and $0 \leq s \leq t \leq b$, there exists a functions $\Psi: \Delta \rightarrow R^{+}$such that $\chi\left(h\left(t, s, D_{1}\right)\right) \leq \Psi(t, s) \chi\left(D_{1}\right)$, where $\sup _{t \in[0, b]} \int_{0}^{t} \Psi(t, s) d s:=\psi^{*}<\infty$.

$\left(\mathrm{H}_{7}\right)$ For each $t \in[0, b], \rho(t,$.$) is measurable on [0, t]$ and $\ell(t)=\sup \{|\rho(t, s)|, 0 \leq s \leq s \leq t\}$ is bounded on $[0, b]$. The map $t \rightarrow \rho_{t}$ is continuous from $[0, \mathrm{~b}]$ to $L^{\infty}([0, b], X)$, here $\rho_{t}(s)=\rho(t, s)$. 
Theorem 3.1: Assume that conditions $\left(\mathrm{H}_{1}\right)-\left(\mathrm{H}_{7}\right)$ hold. Then there exists at least one mild solution for problem (1.1) - (1.3) provided that $Z^{*}=N\left(c+M_{i}+L g_{i}+4 \zeta^{*}\left(1+2 \ell \psi^{*}\right)\right)<1$.

Proof: Define an operator $Q: P C([0, b] ; X) \rightarrow P C([0, b] ; X)$ by

$$
Q u(t)=\left\{\begin{array}{l}
T(t)\left[u_{0}-k(u)\right]+\int_{0}^{t} T(t-s) f\left(s, u(s), \int_{0}^{s} \rho(s, \tau) h(s, \tau, u(\tau)) d \tau\right) d s, t \in\left[0, t_{1}\right], \\
g_{i}(t, u(t)), t \in\left(t_{i}, s_{i}\right], i=1,2, \ldots, m, \\
T\left(t-s_{i}\right) g_{i}\left(s_{i}, u\left(s_{i}\right)\right)+\int_{s_{i}}^{t} T(t-s) f\left(s, u(s), \int_{0}^{s} \rho(s, \tau) h(s, \tau, u(\tau) d \tau) d s, t \in\left(s_{i}, t_{i+1}\right], i=0,1, \ldots, m\right.
\end{array}\right.
$$

for all $u \in P C([0, b] ; X)$, and show that the operator $Q$ satisfies the hypothesis of theorem 2.6.

The proof consists of several steps.

Step1: The operator $Q$ is continuous.

Let $\left\{u_{k}\right\}$ be a sequence such that $u_{k} \rightarrow u$ in $P C([0, b] ; X)$. Then by $\left(\mathrm{H}_{5}\right)$, we have that $f\left(s, u_{k}(s), \int_{0}^{s} \rho(s, \tau) h\left(s, \tau, u_{k}(\tau)\right) d \tau \rightarrow f\left(s, u(s), \int_{0}^{s} \rho(s, \tau) h(s, \tau, u(\tau)) d \tau\right),(k \rightarrow \infty)\right.$ for all $s \in[0, b]$.

Case 1: For each $t \in\left[0, t_{1}\right]$, we have

$$
\begin{gathered}
\left\|\left(Q u_{k}\right)(t)-(Q u)(t)\right\| \leq N\left\|k\left(u_{k}\right)-k(u)\right\|+N \int_{0}^{t} \| f\left(s, u_{k}(s), \int_{0}^{s} \rho(s, \tau) h\left(s, \tau, u_{k}(\tau)\right) d \tau-\right. \\
f\left(s, u(s), \int_{0}^{s} \rho(s, \tau) h(s, \tau, u(\tau)) d \tau \|_{P C} d s\right. \\
\leq N\left\|k\left(u_{k}\right)-k(u)\right\|+N t_{1} \| f\left(s, u_{k}(s), \int_{0}^{s} \rho(s, \tau) h\left(s, \tau, u_{k}(\tau)\right) d \tau-\right. \\
f\left(s, u(s), \int_{0}^{s} \rho(s, \tau) h(s, \tau, u(\tau)) d \tau \|_{P C} d s\right.
\end{gathered}
$$

Case 2: For each $t \in\left(t_{i}, s_{i}\right], i=1,2, \ldots, m$, We have

$$
\left\|\left(Q u_{k}\right)(t)-(Q u)(t)\right\| \leq\left\|g_{i}\left(s_{i}, u_{k}\left(s_{i}\right)\right)-g_{i}\left(s_{i}, u\left(s_{i}\right)\right)\right\|
$$

Case 3: For each $t \in\left(s_{i}, t_{i+1}\right], i=1,2, \ldots, m$, we get

$$
\begin{gathered}
\left\|\left(Q u_{k}\right)(t)-(Q u)(t)\right\| \leq N\left\|g_{i}\left(s_{i}, u_{k}\left(s_{i}\right)\right)-g_{i}\left(s_{i}, u\left(s_{i}\right)\right)\right\|+N \int_{0}^{t} \| f\left(s, u_{k}(s), \int_{0}^{s} \rho(s, \tau) h\left(s, \tau, u_{k}(\tau)\right) d \tau-\right. \\
f\left(s, u(s), \int_{0}^{s} \rho(s, \tau) h(s, \tau, u(\tau)) d \tau \|_{P C} d s\right. \\
\leq N\left\|g_{i}\left(s_{i}, u_{k}\left(s_{i}\right)\right)-g_{i}\left(s_{i}, u\left(s_{i}\right)\right)\right\|+\left(t_{i+1}-s_{i}\right) \| f\left(s, u_{k}(s), \int_{0}^{s} \rho(s, \tau) h\left(s, \tau, u_{k}(\tau)\right) d \tau-\right. \\
f\left(s, u(s), \int_{0}^{s} \rho(s, \tau) h(s, \tau, u(\tau)) d \tau \|_{P C} d s\right.
\end{gathered}
$$

Thus we infer that $\left\|Q u_{k}-Q u\right\|_{P C} \rightarrow 0$ as $k \rightarrow \infty$,

which implies that the mapping $Q$ is continuous on $P C([0, \mathrm{~b}] ; X)$.

Step 2: The operator $Q$ is bounded.

We claim that $Q W \subseteq W$, for any $u \in W \subseteq P C([0, b] ; X)$; by $\left(\mathrm{H}_{3}\right)(\mathrm{i})$, we have

Case 1: Now, for every $t \in\left[0, t_{1}\right]$, we have 
$\|(Q u)(t)\| \leq\|T(t)\|\left\|\left[u_{0}-k(u)\right]\right\|+\int_{0}^{t}\|T(t-s)\| \| f\left(s, u(s), \int_{0}^{s} \rho(s, \tau) h(s, \tau, u(\tau) d \tau) \| d s\right.$

$$
\begin{gathered}
\leq N\left[\left\|u_{0}\right\|+c r+d\right]+N t_{1} m_{f}(s) \Omega_{f}\left[\|u\|+\ell \int_{0}^{s} v(s, \tau) d \tau\|u\|\right] \\
\leq N\left[\left\|u_{0}\right\|+c r+d\right]+N t_{1} m_{f}(s) \Omega_{f}\left(1+\ell v^{*}\right) \\
\left.\leq N\left[\left\|u_{0}\right\|+c r+d\right]+b r m_{f}(s) \Omega_{f}\left(1+\ell v^{*}\right)\right] \\
\leq r
\end{gathered}
$$

Case 2: Now, for every $t \in\left(t_{i}, s_{i}\right], i=1,2, \ldots, m$, we get

$$
\|(Q u)(t)\| \leq\left\|g_{i}\left(s_{i}, u\left(s_{i}\right)\right)\right\| \leq M_{i}\|u\| \leq r
$$

Case 3: Now, for each $t \in\left(s_{i}, t_{i+1}\right], i=1,2, \ldots, m$, we get

$\|(Q u)(t)\| \leq\left\|T\left(t-s_{i}\right)\right\|\left\|g_{i}\left(s_{i}, u\left(s_{i}\right)\right)\right\|+\int_{s_{i}}^{t}\|T(t-s)\|\left\|f\left(s, u(s), \int_{0}^{s} \rho(s, \tau) h(s, \tau, u(\tau)) d \tau\right)\right\| d s$

$$
\begin{gathered}
\leq N M_{i}\|u\|+N\left(t_{i+1}-s_{i}\right) m_{f} \Omega_{f} r\left(1+v^{*} \ell\right) \\
\leq N\left[M_{i} r+b r m_{f} \Omega_{f}\left(1+v^{*} \ell\right)\right] \\
\leq r
\end{gathered}
$$

From above, we have, $Q u \in W$. Which implies that $Q W \subseteq W$.

Step 3: $Q(W)$ is equicontinuous.

Case 1: For interval $\left[0, t_{1}\right], 0 \leq z_{1} \leq z_{2} \leq t_{1}$, and for each $Q \in W(u)$, we have

$$
\begin{gathered}
\left\|(Q u)\left(z_{2}\right)-(Q u)\left(z_{1}\right)\right\| \leq\left\|T\left(z_{2}\right)-T\left(z_{1}\right)\right\|\left\|u_{0}-k(u)\right\| \\
+\| \int_{0}^{z_{2}} T\left(z_{2}-s\right) f\left(s, u(s), \int_{0}^{s} \rho(s, \tau) h(s, \tau, u(\tau)) d \tau\right. \\
-\int_{0}^{z_{1}} T\left(z_{1}-s\right) f\left(s, u(s), \int_{0}^{s} \rho(s, \tau) h(s, \tau, u(\tau)) d \tau \|\right. \\
\leq\left\|T\left(z_{2}\right)-T\left(z_{1}\right)\right\|\left[\left\|u_{0}\right\|+c r+d\right] \\
+\int_{0}^{z_{1}}\left\|T\left(z_{2}-s\right)-T\left(z_{1}-s\right)\right\| m_{f}(s) r \Omega_{f}\left(1+\ell v^{*}\right) d s \\
+\int_{z_{1}}^{z_{2}}\left\|T\left(z_{2}-s\right)\right\| m_{f}(s) r \Omega_{f}\left(1+\ell v^{*}\right) d s
\end{gathered}
$$

Case 2: For interval $\left(t_{i}, s_{i}\right], i=1,2, \ldots, m, t_{i} \leq z_{1} \leq z_{2} \leq s_{i}, u \in W$, we have

$$
\left\|(Q U)\left(z_{2}\right)-(Q u)\left(z_{1}\right)\right\| \leq L_{g_{i}}\left\|u\left(z_{2}\right)-u\left(z_{1}\right)\right\|
$$

Case 3: For interval $\left(s_{i}, t_{i+1}\right], i=1,2, \ldots, m, s_{i} \leq z_{1} \leq z_{2} \leq t_{i+1}, u \in W$ we get

$$
\begin{gathered}
\left\|(Q u)\left(z_{2}\right)-(Q u)\left(z_{1}\right)\right\| \leq\left\|T\left(z_{2}-s_{i}\right)-T\left(z_{1}-s_{i}\right)\right\| L_{g_{i}}\left\|u\left(z_{2}\right)-u\left(z_{1}\right)\right\| \\
+\| \int_{s_{i}}^{z_{2}} T\left(z_{2}-s\right) f\left(s, u(s), \int_{0}^{s} \rho(s, \tau) h(s, \tau, u(\tau)) d \tau\right. \\
-\int_{s_{i}}^{z_{1}} T\left(z_{1}-s\right) f\left(s, u(s), \int_{0}^{s} \rho(s, \tau) h(s, \tau, u(\tau)) d \tau \|\right.
\end{gathered}
$$




$$
\begin{gathered}
\leq\left\|T\left(z_{2}-s_{i}\right)-T\left(z_{1}-s_{i}\right)\right\| L_{g_{i}}\left\|u\left(z_{2}\right)-u\left(z_{1}\right)\right\| \\
+\int_{s_{i}}^{z_{1}}\left\|T\left(z_{2}-s\right)-T\left(z_{1}-s\right)\right\| m_{f} r \Omega_{f}\left(1+v^{*} \ell\right) d s \\
+\int_{z_{1}}^{z_{1}}\left\|T\left(z_{2}-s\right)\right\| m_{f} r \Omega_{f}\left(1+v^{*} \ell\right) d s
\end{gathered}
$$

From above, we get

$$
\left\|(Q u)\left(z_{2}\right)-(Q u)\left(z_{1}\right)\right\| \rightarrow 0 \text { as } z_{2} \rightarrow z_{1} \text {. So Q is equicontinuous. }
$$

Step 4: Mönch condition holds.

Suppose that $V \subseteq W$ is countable and $V \subseteq \overline{\operatorname{conv}}(\{0\} \cup Q(V))$. We show that $\chi(V)=0$ where $\chi$ is the Hausdroff measure of noncompactness. Without loss of generality, we may assume that $V=\left\{u_{k}\right\}_{k=1}^{\infty}$ and we can easily verify that $V$ is bounded and equicontinuous.

Case 1: For each $t \in\left[0, t_{1}\right]$ we get

$$
\begin{gathered}
(Q u)(t)=T(t)\left[u_{0}-k(u)\right]+\int_{0}^{t} T(t-s) f\left(s, u(s), \int_{0}^{s} \rho(s, \tau) h(s, \tau, u(\tau)) d \tau\right) d s \\
(Q u)(t)=\left(Q_{1} u\right)(t)+\left(Q_{2} u\right)(t) \text { with } \\
\left(Q_{1} u\right)(t)=T(t)\left[u_{0}-k(u)\right] \\
\left(Q_{2} u\right)(t)=\int_{0}^{t} T(t-s) f\left(s, u(s), \int_{0}^{s} \rho(s, \tau) h(s, \tau, u(\tau)) d \tau\right) d s
\end{gathered}
$$

Moreover, $Q_{1}: V \rightarrow P C([0, b] ; X)$ is Lipschitz continuous with constant $N c$ due to the conditions $\left(\mathrm{H}_{1}\right)$ and $\left(\mathrm{H}_{4}\right)$. In fact $u, v \in V$, we have

$$
\begin{gathered}
\left\|\left(Q_{1} u\right)(t)-\left(Q_{1} v\right)(t)\right\|=\sup _{t \in\left[0, t_{1}\right]}\|T(t)[k(u)-k(v)]\| \\
\leq N c\|u-v\|_{P C}
\end{gathered}
$$

So, from lemma $2.1-2.3,2.5$ and hypotheses $\left(\mathrm{H}_{3}\right)(\mathrm{ii}),\left(\mathrm{H}_{6}\right)(\mathrm{ii})$, we have

$$
\begin{gathered}
\chi\left(\left\{Q u_{k}\right\}_{k=1}^{\infty}\right) \leq \chi\left(\left\{Q_{1} u_{k}\right\}_{k=1}^{\infty}\right)+\chi\left(\left\{Q_{2} u_{k}\right\}_{k=1}^{\infty}\right) \\
\leq N c \chi\left(\left\{u_{k}\right\}_{k=1}^{\infty}\right)+\chi\left(\int_{0}^{t} T(t-s) f\left(s, u(s), \int_{0}^{s} \rho(s, \tau) h(s, \tau, u(\tau)) d \tau\right) d s\right) \\
\leq N c \chi\left(\left\{u_{k}\right\}_{k=1}^{\infty}\right)+2 N \int_{0}^{t} \chi\left(f\left(s, u(s), \int_{0}^{s} \rho(s, \tau) h(s, \tau, u(\tau)) d \tau\right) d s\right) \\
\leq N c \chi\left(\left\{u_{k}\right\}_{k=1}^{\infty}\right)+2 N \int_{0}^{t} \eta(s)\left(\sup _{0 \leq s \leq t_{1}} \chi\left(\left\{u_{k}(s)\right\}_{k=1}^{\infty}\right)+\chi\left(\int_{0}^{s} \rho(s, \tau) h\left(s, \tau,\left\{u_{k}(\tau)\right\}_{k=1}^{\infty}\right) d \tau\right)\right) d s \\
\leq N c \chi\left(\left\{u_{k}\right\}_{k=1}^{\infty}\right)+2 N \int_{0}^{t} \eta(s)\left(\sup _{0 \leq s \leq t_{1}} \chi\left(\left\{u_{k}(s)\right\}_{k=1}^{\infty}\right)+2 \ell \int_{0}^{s} \psi(s, \tau) d \tau \chi\left(\left\{u_{k}(\tau)\right\}_{k=1}^{\infty}\right)\right) d s \\
\leq N c \chi\left(\left\{u_{k}\right\}_{k=1}^{\infty}\right)+2 N \int_{0}^{t} \eta(s)\left(\sup _{0 \leq s \leq t_{1}} \chi\left(\left\{u_{k}(s)\right\}_{k=1}^{\infty}\right)+2 \ell \psi^{*} \chi\left(\left\{u_{k}(\tau)\right\}_{k=1}^{\infty}\right)\right) d s \\
\leq N c \chi\left(\left\{u_{k}\right\}_{k=1}^{\infty}\right)+2 N \zeta^{*}\left(1+2 \ell \psi^{*}\right)\left(\sup _{0 \leq s \leq t_{1}} \chi_{P C}(V(s))\right) \leq N\left(c+2 \zeta^{*}\left(1+2 \ell \psi^{*}\right)\right) \sup _{0 \leq s \leq t_{1}} \chi_{P C}(V(s))
\end{gathered}
$$

Case 2: For each $t \in\left(t_{i}, s_{i}\right], i=1,2, \ldots, m$, we have 
Existence Results of Abstract Impulsive Integrodifferential Systems with Measure of Non-compactness

$$
\begin{gathered}
\chi\left(\left\{Q u_{k}\right\}_{k=1}^{\infty}\right) \leq \chi\left(g_{i}\left(s_{i}, u\left(s_{i}\right)\right)\right. \\
\leq M_{i} \sup _{t_{i} \leq s \leq s_{i}} \chi_{P C}(V(s))
\end{gathered}
$$

Case 3: For each $t \in\left(s_{i}, t_{i+1}\right], i=1,2, \ldots, m$, we get

$$
\begin{gathered}
(Q u)(t)=T\left(t-s_{i}\right) g_{i}\left(s_{i}, u\left(s_{i}\right)\right)+\int_{0}^{t} T(t-s) f\left(s, u(s), \int_{0}^{s} \rho(s, \tau) h(s, \tau, u(\tau)) d \tau\right) d s \\
(Q u)(t)=\left(Q_{1} u\right)(t)+\left(Q_{2} u\right)(t) \text { with } \\
\left(Q_{1} u\right)(t)=T\left(t-s_{i}\right) g_{i}\left(s_{i}, u\left(s_{i}\right)\right) \\
\left(Q_{2} u\right)(t)=\int_{0}^{t} T(t-s) f\left(s, u(s), \int_{0}^{s} \rho(s, \tau) h(s, \tau, u(\tau)) d \tau\right) d s
\end{gathered}
$$

Moreover, $Q_{1}: V \rightarrow P C([0, b] ; X)$ is Lipschitz continuous with constant $N L g_{i}$ due to the conditions $\left(\mathrm{H}_{1}\right)$ and $\left(\mathrm{H}_{2}\right)(\mathrm{i})$. In fact $u, v \in V$, we have

$$
\begin{aligned}
\left\|\left(Q_{1} u\right)(t)-\left(Q_{1} v\right)(t)\right\| & =\left\|T\left(t-s_{i}\right)\right\|\left\|g_{i}\left(s_{i}, u\left(s_{i}\right)\right)-g_{i}\left(s_{i}, v\left(s_{i}\right)\right)\right\| \\
& \leq N L g_{i}\|u-v\|_{P C}
\end{aligned}
$$

So, from lemma $2.1 \quad-2.3, \quad 2.5$ and hypotheses $\left(\mathrm{H}_{3}\right)(\mathrm{ii}), \quad\left(\mathrm{H}_{6}\right)(\mathrm{ii})$, we have $\chi\left(\left\{Q u_{k}\right\}_{k=1}^{\infty}\right) \leq \chi\left(\left\{Q_{1} u_{k}\right\}_{k=1}^{\infty}\right)+\chi\left(\left\{Q_{2} u_{k}\right\}_{k=1}^{\infty}\right)$

$$
\begin{gathered}
\leq N L g_{i} \chi\left(\left\{u_{k}\right\}_{k=1}^{\infty}\right)+\chi\left(\int_{s_{i}}^{t} T\left(t-s_{i}\right) f\left(s, u(s), \int_{0}^{s} \rho(s, \tau) h(s, \tau, u(\tau)) d \tau\right) d s\right) \\
\leq N L g_{i} \chi\left(\left\{u_{k}\right\}_{k=1}^{\infty}\right)+2 N \int_{s_{i}}^{t} \chi\left(f\left(s, u(s), \int_{0}^{s} \rho(s, \tau) h(s, \tau, u(\tau)) d \tau\right) d s\right) \\
\leq N L g_{i} \chi\left(\left\{u_{k}\right\}_{k=1}^{\infty}\right)+2 N \int_{s_{i}}^{t} \eta(s)\left(\sup _{s_{i} \leq s \leq t_{i+1}} \chi\left(\left\{u_{k}(s)\right\}_{k=1}^{\infty}\right)+2 \ell \int_{0}^{s} \psi(s, \tau) d \tau \chi\left(\left\{u_{k}(\tau)\right\}_{k=1}^{\infty}\right)\right) d s \\
\leq N L g_{i} \chi\left(\left\{u_{k}\right\}_{k=1}^{\infty}\right)+2 N \int_{s_{i}}^{t} \eta(s)\left(\sup _{s_{i} \leq s \leq t_{i+1}} \chi\left(\left\{u_{k}(s)\right\}_{k=1}^{\infty}\right)+2 \ell \psi^{*} \chi\left(\left\{u_{k}(\tau)\right\}_{k=1}^{\infty}\right)\right) d s \\
\leq N\left(L g_{i}+2 \zeta^{*}\left(1+2 \ell \psi^{*}\right)\right) \sup _{s_{i} \leq s \leq t_{i+1}} \chi_{P C}(V(s))
\end{gathered}
$$

From above, we have

$$
\begin{gathered}
\chi_{P C}(Q(V)) \leq N\left(c+M_{i}+L g_{i}+4 \zeta^{*}\left(1+2 \ell \psi^{*}\right)\right) \chi_{P C}(V) \\
\leq Z^{*} \chi_{P C}(V), \text { where } Z^{*}=N\left(c+M_{i}+L g_{i}+4 \zeta^{*}\left(1+2 \ell \psi^{*}\right)\right)<1
\end{gathered}
$$

Thus from Mönch condition we get

$$
\begin{gathered}
\chi_{P C}(V) \leq \chi_{P C}(\overline{\operatorname{conv}}\{0\} \cup Q(V))=\chi_{P C}(Q(V)) \leq Z^{*} \chi_{P C}(V) \text { which implies that } \\
\chi_{P C}(V)=0
\end{gathered}
$$

Hence using Theorem 2.6, there is a fixed point of $u$ of $Q$ in $W$. Which is a mild solution of (1.1) -(1.3). This completes the proof.

\section{Example}

In this section, $X=L^{2}([0, \pi], R)$ and define the operator $A$ by $A x=x^{\prime \prime}$ with the domain 
$D(A)=\left\{x \in X: x^{\prime \prime} \in X, x(0)=x(\pi)=0\right\}$. It is well known that $A$ is the bounded linear operator of a compact semigroup $\mathrm{T}(t), t \geq 0$ on $\mathrm{X}$ and that $\|\mathrm{T}(t)\| \leq e^{-t}$ for all $t \geq 0$.

Consider a nonlocal problem of impulsive integrodifferential equations given by,

$$
\begin{gathered}
\frac{\partial u(t, w)}{\partial t}=\frac{\partial^{2} u(t, w)}{\partial w^{2}} u(t, w)+\int_{0}^{t} h(t, s, u(s, w)) d s+F(t, u(t, w)), \\
(t, w) \in \bigcup_{i=1}^{m}\left[s_{i} t_{i+1}\right] \times[0, \pi] \\
u(t, 0)=u(t, \pi)=0, t \in[0, b] \\
u(0, w)+\sum_{j=1}^{m} c_{j} u\left(t_{j}, w\right)=u_{0}(w), w \in[0, \pi] \\
u(t, w)=G_{i}(t, u(t, w)), w \in[0, \pi], t \in\left(t_{i}, s_{i}\right]
\end{gathered}
$$

with $0=t_{0}=s_{0}<t_{1} \leq s_{1} \leq t_{2}<\ldots .<t_{m} \leq s_{m} \leq t_{m+1}=b$ are fixed real numbers, $u_{0} \in X, F \in C([0, b] \times R ; R)$ and $G_{i} \in C\left(\left(t_{i}, s_{i}\right] \times R ; R\right)$ for all $i=1,2, \ldots, m$.

To represent the problem (4.1) - (4.4) in the abstract form (1.1) - (1.3), we assume that

(i). $\quad f:[0, b] \times X \rightarrow X$ defined by $f(t, x)(w)=\int_{0}^{t} h(t, s, x(w)) d s+F(t, x(w))$ for $t \in[0, b], w \in[o, \pi]$

(ii). $\quad k: P C([0, b] ; X) \rightarrow X$ is continuous function defined by $k(u)(w)=u_{0}(w)-\sum_{i=1}^{m} c_{j} u\left(t_{j}\right)(w)$,

$$
t \in[0, b], w \in[0, \pi], \text { where } u(t)(w)=u(t, w), t \geq 0, w \in[0, \pi]
$$

(iii). $\quad g_{i}:\left(t_{i}, s_{i}\right] \times X \rightarrow X$ defined by $g_{i}(t, x)(w)=G_{i}(t, x(w))$.

Now, we say that $u \in P C(X)$ is a mild solution of (4.1)-(4.4) if $u($.$) is a mild solution of the associated$ abstract problem (1.1)-(1.3).

\section{Acknowledgement}

The first author would like to acknowledgement the financial aid from University Grants Commission, Hyderabad, under the research project, MRP-4921/14(SERO/UGC)

\section{References}

Ntouyas, S.K., and Tsamatos,P., Ch.: Global existence for second order semilinear ordinary and delay integro differential with nonlocal conditions, Applicable Anal.Vol.67, no. 3-4, pp. 245-257, 1997.

Dong, Q., Li, G.: Existence of solutions for semilinear differential equations with nonlocal conditions in Banach spaces, Electron.J.Qual.Theory Differ. Equ. 2009, 47 (2009)

Liang, J., Liu, J.H., Xiao, T.J.: Nonlocal Cauchy problems governed by compact operator families, Nonlinear Anal. 57 (2004) 183-189

Mallika Arjunan,.M,Kavitha,.V.,Selvi,.S.:Existence results for impulsive differential equations with nonlocal conditions via measures of noncompactness, J.Nonlinear Sci.Appl., Vol. 5 (2012), 195-205 
A.Pazy.: Semigroups of linear operators and Applications to partial differential equations, Springer-Verlg, Newyork, (1983)

Malar,.K, and Tamilarasi, A.: Existence of mild solutions for nonlocal impulsive a integrodifferential equations with the measure of noncompactness, Far East Journal of Applied Mathematics, Vol.57, no.1, pp.15-31, 2011

Ahmad, B., Malar,. K, and Karthikeyan, K.: A study of nonlocal problems of impulsive integrodifferential equations with measure of noncompactness, Advances in Difference equations, Vol.2013, article 205, 2013

Wang,J., Zhou,Y., Fecckan,M.: On recent developments in the theory of boundary value problems for impulsive fractional differential equations, Comput. Math. Appl. 64, 3008-3020 (2012)

Lan,HY., Cui, YS.: On the existence of solutions for nonlinear first-order implicit impulsive integro-differential equations, Nonlinear Anal. 71(5-6), 1670-1677 (2009)

Nieto,J., O'Regan, D.: Variational approach to impulsive differential equations, Nonlinear Anal. RWA 10, 680-690 (2009)

Yang, Y., Wang, J.: On Some existence results of mild solutions for nonlocal integrodifferential Cauchy problems in Banach spaces, Opuscula mathematics, Vol. 31, no. 3, pp. 443-455, 2011

Lizama, C.,Pozo, J., C.: Existence of mild solutions for a semilinear integrodifferential equation with nonlocal initial conditions, Abstract and Applied Analysis, vol. 2012, Article ID 647103, 15 pages 2012

Anguraj, A.,Mallika Arjunan, M.: Existence results foe for an impulsive neutral integrodifferential equations in Banach spaces, Nonlinear studies, Vol. 16(1), 33-48, (2009)

Shaochun, J., Gang, L.: A unified approach to nonlocal impulsive differential equations with the measure of noncompactness, Advances in Difference equations, Vol. 2012, 182, (2012)

Hernandez, E., O’Regan, D.: On a new class of abstract impulsive differential equations Proc. Am. Math, Soc. 141, 1641-1649 (2013)

Wang., J, Li,: Periodic BVP integer/fractional order nonlinear differential equations with non-instantaneous impulses J. Appl. Math. comput, DOI 10.1007/s 12190-013-0751-4 (2014)

Banas., J, Goebel, K.: Measure of noncompactness in Banach space Lecture Notes in Pure and Applied Mathematics, Vol. 60, Marcel Dekker, New york (1980)

Mönch., H.: Boundary value problems for nonlinear ordinary differential equations of second order in Banach spaces Nonlinear Anal. 4, 985-999 (1980)

Xue.,X.: Semilinear nonlocal differential equations with measure of noncompactness in Banach spaces J.Nanjing Univ. Mayh. Big. 24, 264-276 (2007)

Bothe., D.: Multivalued perturbation of m-accretive differential inclusions lsr. J Math. 108. 109-138 (1998) 